\title{
Patellar clonus: an autonomous central generator
}

\author{
E G WALSH, G W WRIGHT \\ From the Department of Physiology, Edinburgh University, Edinburgh, UK
}

SUMMARY The mechanisms underlying clonus are considered in relation to current theories. By the use of a lever attached to a printed motor forces have been applied to the upper edge of the patella, a steady stretching force in suitable hemiplegic patients started the clonic oscillations. With rhythmic forces it has been found that the underlying rhythm is very resistent to entrainment. The oscillation provoked by a steady bias is increased in amplitude but unchanged in frequency when inertia is added to the apparatus. The frequency of clonus at the ankle, patella and wrist is similar. These and other considerations lead to the conclusion that clonus is not due to the self re-excitation of stretch reflexes but to an autonomous central generator.

The view that clonus is due to the self re-excitation of hyperactive stretch reflexes (feedback oscillator theory) has recently been reiterated. ${ }^{12}$ Should this be correct the pulsatile rather than sinusoidal nature of the discharges indicates high non-linearity, but observations on patellar clonus have shown that the rhythm is generated by an autonomous central generator. A similar conclusion was reached in studies of ankle clonus. ${ }^{3}$ Three sets of observations are relevant:

\section{Failure to entrain}

The study of the effects of rhythmic inputs on oscillators has a long history, several distinguished scientists have been interested in this problem. ${ }^{4}$ Huygens (1629-1695) noted that two clocks mounted in the same board came into step. Lord Rayleigh found that two electrical tuning forks synchronised when fed from the same battery and observed a similar phenomenon in two organ pipes fed from the same wind chest. In these examples the transfer of tiny amounts of energy led to "entrainment", one oscillator influencing another. Sir Edward Appleton ${ }^{5}$ made a study of the mathematics underlying the synchronisation of a valve oscillator by powerful distant transmitter. Dr A Linnett (Department of Engineering, Edinburgh University) has rein-

Address for reprint requests: Dr E G Walsh, Department of Physiology, University Medical School, Teriot Place, Edinburgh EH89AG, UK.

Received 5 December 1986 and in revised form 4 February 1987. Accepted 11 February 1987 vestigated the entrainment of feedback oscillators. All such systems are non-linear, otherwise the amplitude of oscillation would be infinite, but there are different types and degrees of non-linearity. One model had non-linear damping and another a non-linear spring. On an analogue computer both systems entrained when excited over a certain frequency range; outside of this beats were set up. As would be expected the more powerful the entraining rhythm the wider the frequency range over which entrainment occurs. How wide the entrainment zone is can only be predicted by a rigorous mathematical analysis of the particular system under consideration. Stein et $^{a^{6}}{ }^{6}$ also obtained beats with a feedback oscillator but they do not appear to have considered the possibility of entrainment with closer frequencies.

Two hemiplegic patients with patellar clonus were studied whilst lying on a couch. Steady bias provided by a padded bar connected to a printed motor stretched the quadriceps and initiated the clonus. When a rhythmic component was added to the bias entrainment was never seen (fig a). Beating does not necessarily exclude a feedback oscillator but it occurred with frequencies as close as $0.25 \mathrm{~Hz}$ when the force was about the same as that produced by the quadriceps. These findings indicate that the rhythm is generated by a system which is quite insensitive to peripheral conditions and the behaviour is quite different from the feedback oscillators that were modelled. Similar observations have been made with the ankle and wrist clonus ${ }^{3}$ and are difficult to explain unless the rhythm is generated centrally and not dependent on cycle by cycle feedback. 


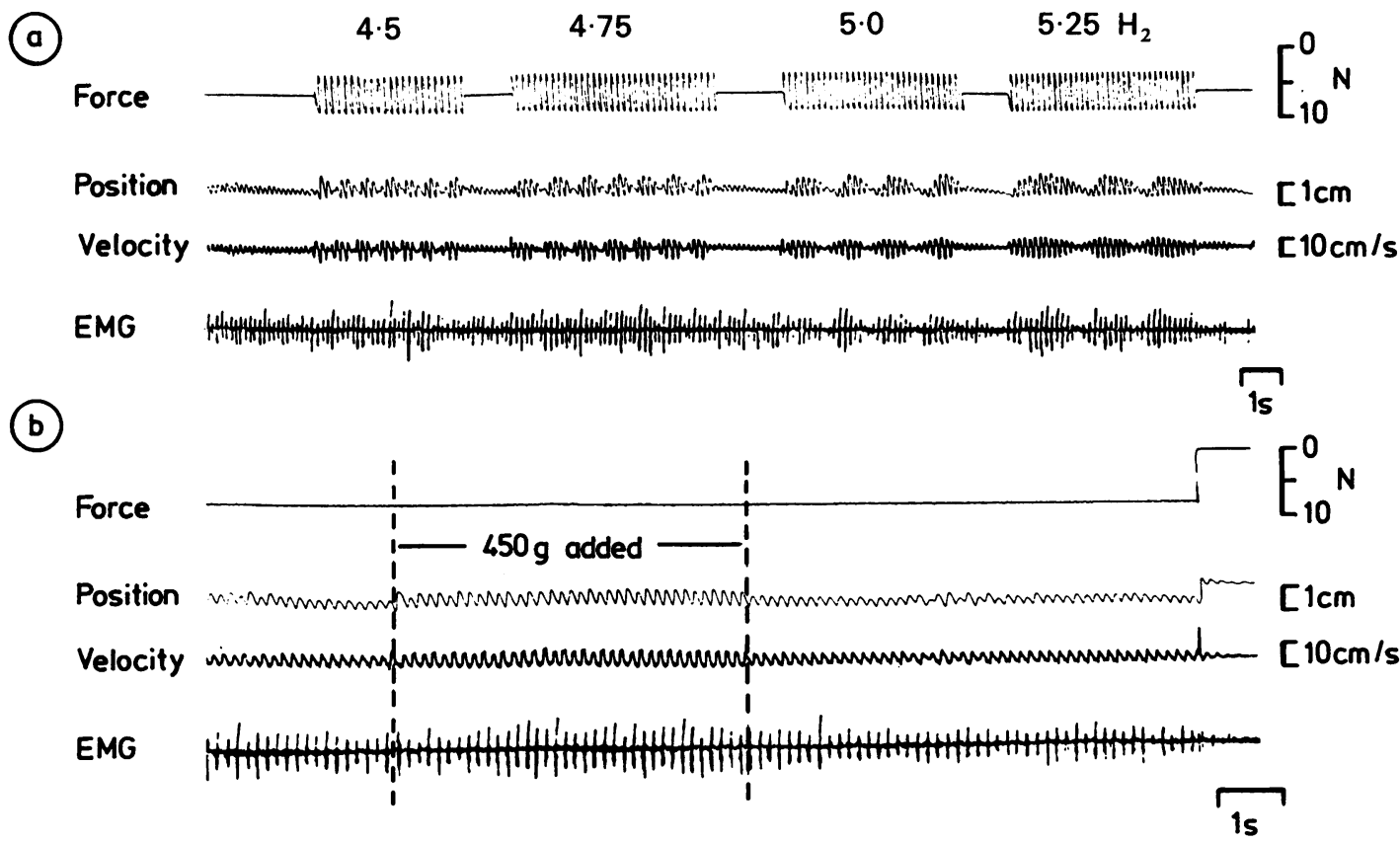

Fig (a) Relationship between force and motion of the patella. A steady bias stretches the quadriceps throughout and on four occasions a rhythmic component of force is also introduced at the indicated frequencies. Beats are set up at rates corresponding to the difference in frequency between the applied force and that of the clonus. (b) The effect of adding mass to the system is to increase the amplitude of oscillation. When the bias is switched off the clonus peters out. Woman aged 47 with dense left hemiplegia following subarachnoid haemorrhage.

\section{Increased amplitude with added inertia}

With the motor providing steady bias weights of up to $500 \mathrm{~g}$ were added to the lever which moved in the horizontal plane. The effect was to increase the amplitude of the oscillation (fig b). There was no systematic change of frequency. These findings are readily explained if the clonus is generated by an autonomous central generator; evidently the extra mass tuned the system mechanically to a resonant frequency closer to the rhythm generator frequency. The findings are inconsistent with the feedback oscillator theory.

\section{Frequency of the clonus}

It has been claimed that patellar clonus is more rapid

Table Frequency of clonus

\begin{tabular}{llll}
\hline & Ankle & Patella & Wrist \\
\hline Iansek $^{1}$ & $6.4 \pm 0.8(21)$ & $7 \cdot 5,7 \cdot 8,10$ & $8 \cdot 5 \pm 0.8(5)$ \\
This laboratory $^{2}$ & $6.0 \pm 1 \cdot 2(7)$ & $6 \cdot 0,6 \cdot 25,6.5$ & $5 \cdot 5 \pm 0.4(6)$ \\
\hline
\end{tabular}

Means and standard deviations for ankle and wrist clonus. Figures in brackets are number of patients. Individual values are given for the measurements of patella clonus. than ankle clonus and slower than wrist clonus. ${ }^{1}$ These differences are said to support the feedback hypothesis and to be due to the different lengths of the nerves concerned. The results need reconsideration and relevant data are summarised in the table. In this laboratory seven patients with ankle clonus have been studied, the clonus being sustained by a continuous dorsiflexing torque from a G16M4 printed motor. Six patients with wrist clonus have also been studied, a torque from G9M4 motor being used to extend the joint. Of the three values for patellar clonus given by Iansek ${ }^{1}$ two are less than two standard deviations from the mean of his value for ankle clonus. The claim that wrist clonus is unusually rapid receives no support from the reinvestigation that has now been undertaken. The rate at the wrist and the ankle in one patient was identical $(5.0 \mathrm{~Hz})$. The data contradict the feedback hypothesis.

\section{Discussion}

The frequency of clonus in any one patient is remarkably constant over long periods and remains unchanged with different postures; changes in the 
mechanical conditions alter the amplitude of clonus but not its frequency. Cooling the limb, impeding the motion with a viscous solution and mechanical obstruction do not affect frequency. ${ }^{7}$ When by artificial means a limb is made to form part of a feedback oscillator the frequency varies widely with peripheral conditions. ${ }^{8}$ During general or spinal anaesthesia clonus may still be elicited when tendon jerks are abolished (JAV Bates, personal communication). The finding that spindle afferents discharge during clonus on the falling phase of muscular contraction ${ }^{9}$ in no way negates the view that the system is driven centrally.

In some experiments weights and springs have been attached to limbs hoping thereby to elucidate the problem of clonus, ${ }^{2}$ but certain engineering principles must be considered for the sharpness of tuning; $Q$, of a mechanical system is given by the equation:

$$
\mathrm{Q}=\frac{1}{\mathrm{c}} \sqrt{\mathrm{K} \cdot \mathrm{J}}
$$

where $\mathrm{c}$ is the damping coefficient, $\mathrm{K}$ the stiffness and $\mathbf{J}$ the inertia. By adding masses and springs $\mathbf{Q}$ will rise; the tendency to resonate will increase. Reflex effects will thus be accentuated to an abnormal degree. Feedback oscillation may indeed be set up which however does not bear on the problem of clonus; indeed such a system may set up tremor in a hitherto stable postural system. The lack of entrainment, the effect of added inertia and a consideration of the rates of oscillation all point to a central generator.

\section{References}

1 Iansek R. The effects of reflex path length on clonus frequency in spastic muscles. J Neurol Neurosurg Psychiatry 1984;47:1122-4.

2 Rack PMH, Ross HF. Thilmann AF. The ankle stretch reflexes in normal and spastic subjects. Brain 1984;107:637-54.

3 Walsh EG. Clonus: beats provoked by the application of a rhythmic force. J Neurol Neurosurg Psychiatry 1976;39:266-74.

4 Minorsky N. Non-linear Oscillations. Princeton: van Nostrand Co Inc NJ, 1962.

5 Appleton EV. The automatic synchronisation of triode scillators. Proc Cambridge Philosophical Society 1922;21:231-48.

6 Stein RB, Gordon T, Oguztoreli MN, Lee RG. Classifying sensory patterns and their effects on locomotion and tremor. Can J Physiol Pharmacol 1981;59:645-55.

7 Dimitrijevic MR, Nathan PW, Sherwood AM. Clonus: the role of central mechanisms. J Neurol Neurosurg Psychiatry 1980;43:321-32.

8 Walsh EG. Tremor of the wrist induced by positive velocity feedback. J Physiol (Lond) 1970;207:16-7.

9 Hagbarth KE, Wallin G, Löfstedt L. Aquilonius SM. Muscle spindle activity in alternating tremor of Parkinsonism and in clonus. $J$ Neurol Neurosurg Psychiatry 1975;38:636-41. 